\title{
Arbor
}

\section{Actitudes hacia la influencia de la sociedad en la ciencia y la tecnología}

\author{
M. ${ }^{a}$ Antonia Manassero Mas y Ángel Vázquez Alonso
}

Arbor CLXII, 637 (Enero 1999), 45-72 pp.

La filosofía tradicional de la ciencia presenta ésta como un conoci. miento objetivo, aislado de influencias externas, mientras que la moderna sociología y epistemología de la ciencia sostienen la influencia de los factores contextuales. Este estudio analiza las actitudes de estudiantes y profesorado sobre la influencia de la sociedad en la ciencia y tecnología mediante una serie de cuestiones referidas a distintos factores sociales, como el gobierno, las empresas e industrias, el ejército, la cultura, los grupos de especial interés o la educación científica y tecnológica del público. Las actitudes obtenidas muestran diferente intensidad y se fundan en distintas razones justificativas, aunque se observa una tendencia a admitir mayoritariamente esta influencia sobre la ciencia cuando se refiere a un factor general, mientras que cuando la influencia de la sociedad se concreta en factores más concretos, las actitudes no concitan tanta unanimidad. Las diferencias entre estudiantes según el grado de exposición a la ciencia no son tan importantes como cabría esperar, y las diferencias de género, cuando se controla la variable anterior son escasas. El análisis comparativo entre profesorado y alumnado muestra más coincidencias que diferencias, y cuando se comparan los estudiantes canadienses con los españoles existen tantas diferencias como coincidencias. Finalmente, se discuten algunas implicaciones para la enseñanza de las ciencias desde una perspectiva de ciencia-tecnología-sociedad (CTS). 
El positivismo lógico propugna una visión de la ciencia acumulativa, objetiva y neutral, basada en una estricta fenomenología empirista, aislada e impermeable a las influencias externas y ha sido considerada como la filosofía de la ciencia por antonomasia. El positivismo ha sido criticado intensamente desde posiciones sociológicas y epistemológicas surgidas en el segundo tercio del siglo XX, como el relativismo, el realismo o el funcionalismo, fundadas en análisis históricos y sociológicos de la ciencia; en particular, la ausencia de las influencias en la génesis del conocimiento científico, ajenas a los propios hechos empíricos (psicológicas, sociales, etc.) es una tesis positivista imposible de sostener. Incluso el neopositivismo ha suavizado algunas de las tesis más duras del positivismo lógico en relación con la interacción de la ciencia con la sociedad, diferenciando entre contextos de descubrimiento y contextos de justificación (Reichenbach, 1966). El conocimiento científico y tecnológico es una construcción social humana, y como tal, no resulta ajeno a ninguno de los condicionantes habituales de las actividades humanas, aunque uno de sus logros más importantes es maximizar la racionalidad en el conocimiento y minimizar los sesgos propios de la condición humana. La realidad es que ciencia, tecnología y sociedad son tres patas de un mismo edificio cuya interacción mutua y bidireccional es esencial para comprender su estatus actual; la sociedad influye en el desarrollo de ciencia y tecnología, y la ciencia y tecnología contribuyen significativamente a modelar el desarrollo social (Bernal, 1979; Knorr-Cetina, 1981; Latour y Woolgar, 1996).

A la luz de los conocimientos históricos, epistemológicos y, especialmente, sociológicos se demuestra que ciencia y tecnología son fenómenos sociales, con una dimensión sociológica externa y otra dimensión interna. La dimensión interna de la ciencia se centra en la sociología de la denominada comunidad científica, es decir, las normas, usos, valores y costumbres que rigen el comportamiento dentro de la comunidad científica como grupo (Merton, 1977; Mitroff, 1974). La dimensión externa se centra en las relaciones establecidas entre la comunidad científica y el resto del entramado social, el gobierno, los militares, la industria, el sistema económico-productivo, y la sociedad en general. La comunidad cientifica busca en las instituciones sociales financiación, justificación y apoyo para sus proyectos, mientras que las instituciones sociales obtienen de la comunidad científica progreso en el conocimiento, soluciones a problemas sociales (sanidad, aplicaciones tecnológicas, transportes ...) y, en general, mejora de la calidad de vida. Esta interacción mutua da lugar a una influencia bidireccional, en los dos sentidos: la sociedad influye sobre ciencia y tecnología, y 
el sistema de ciencia y tecnología influye sobre la sociedad (Lamo, González y Torres, 1994).

Por otro lado, ciencia y tecnología son dos realidades cada vez más imbricadas, de modo que muchos tratadistas hablan ya de un complejo denominada tecnociencia donde los límites entre una ciencia y una tecnología clásicas se han borrado bastante de modo que ambas comparten características atribuidas a la otra. Muchos países desarrollados están crecientemente implicados con la toma de decisiones en asuntos sociotécnicos tales como preservación del medio ambiente, gestión de residuos, energías, calentamiento global, biotecnología, etc. Esto ha generado una preocupación por la alfabetización científica, es decir, un conocimiento básico de la ciencia entre el gran público que capacite a las personas para participar en la toma responsable de decisiones y evite la división social entre las dos culturas (científicos-no científicos), que puede conducir al cientifismo o a la tecnocracia. No se trata de que el público sepa mucha ciencia, sino que sepa lo necesario sobre ciencia para poder desenvolverse como ciudadanos en un entorno vital crecientemente tecnologizado. Como muestra paralela de esta preocupación, se han desarrollado esfuerzos demoscópicos para la evaluación del conocimiento e imagen de ciencia y tecnología entre el público (Durant, Evans y Thomas, 1989; Lucas, 1994; Miller, 1983, 1992), y en España se puede citar el informe CIRES (1992) y el CIS (Atienza y Luján,1997). Miller (1983) identifica el 18\% de "público atento", Durant et al. (1989) obtienen el 38\% que se declaran "muy interesados" en ciencia, Lucas (1994) obtiene 17\% muy interesados y $49 \%$ bastante interesados, mientras el CIS (1997) identifica $63 \%$ de personas con mucho y bastante interés en descubrimientos científicos y avances tecnológicos, llegando al $80 \%$ en el caso de los avances médicos. Además las relaciones obtenidas empíricamente entre conocimiento e interés de la gente en la ciencia son positivas (los más interesados en la ciencia superan en conocimientos a los menos interesados). En suma, con independencia del país, más de dos tercios de las personas se declaran interesadas en ciencia, y estos resultados demuestran hasta qué punto la influencia entre ciencia y tecnología y sociedad tienen un interés central para la alfabetización científica.

El propósito de este informe es analizar las opiniones y actitudes de una muestra amplia de estudiantes y profesorado de todos las edades y niveles educativos (desde secundaria a la universidad) sobre la influencia de la sociedad sobre ciencia y tecnología (sociología externa). Esta influencia se ha concretado en algunos sujetos específicos como la influencia del gobierno, el ejército, las industrias, las escuelas, la 
M. ${ }^{a}$ Antonia Manassero Mas y Ángel Vázquez Alonso

cultura y los grupos de especial interés sobre la ciencia y tecnología. Sobre estos temas se ha encuestado a dos muestras de participantes, representantes de jóvenes estudiantes y profesorado.

\section{Método}

\section{Instrumento}

Las cuestiones aplicadas se refieren a los siguientes temas de influencia de la sociedad sobre ciencia y tecnología: dos cuestiones directas sobre la influencia de la sociedad sobre ciencia y tecnología respectivamente (20811, 20821), subvención de la investigación por el gobierno (20111), control de la investigación por las empresas (20211), investigación para fines militares (20311), influencia de la cultura sobre ciencia y tecnología (20411), conocimiento sobre ciencia y tecnología (20521) e influencia de los grupos de especial interés (20611). Estas cuestiones han sido adaptadas de Aikenhead, Ryan y Fleming (1989), las seis últimas, y Rubba y Harkness (1993), las dos primeras, que forman parte de un banco de cuestiones aplicadas en un estudio más amplio (Vázquez y Manassero, 1997), conservando el número de identificación de cinco cifras. En la cuestión 20521 se ha prescindido de una alternativa que comparaba la investigación para fines militares e industriales entre USA y Canadá.

Todas las cuestiones tienen un formato de elección múltiple, que se inicia con un pie de unas pocas líneas, donde se plantea el problema sobre el cual se desea conocer la opinión del encuestado, seguido de una lista de alternativas, cada una de ellas identificada correlativamente con una letra, que ofrecen todo una abanico de diferentes opiniones sobre el tema planteado. En algunos casos, cuando la lista de opiniones alternativas es larga, éstas se ofrecen agrupadas, para facilitar la lectura, la comprensión y la elección, mediante la inclusión de alguna frase intermedia, que divide en grupos el abanico completo de alternativas, por ejemplo, las que están a favor y las que están en contra. Además, todas las cuestiones finalizan con tres opciones comunes que recogen diversas razones para no elegir, y que son las siguientes: 1.No entiendo la cuestión, 2.No sé lo suficiente sobre el tema para seleccionar una opción, y 3.Ninguna de las opciones satisface básicamente mi opinión.

En el estudio original el banco de cuestiones total se estructuró en seis cuestionarios diferentes, de modo que las cuestiones estudiadas 
aquí, pertenecían a diferentes cuestionarios, y por tanto han sido respondidas por muestras diferentes, aunque equivalentes entre sí por el sistema de aplicación balanceada de los cuestionarios. Debido a las limitaciones de espacio no se pueden ofrecer tabulados todos los detalles de los resultados que pueden consultarse en otro lugar (Vázquez y Manassero, 1997). En el caso del profesorado, sólo se contestaron cuatro de las cuestiones citadas (20111, 20211, 20311, 20611).

Finalmente, es importante destacar que las cuestiones aplicadas constituyen una nueva forma de prueba de opción múltiple «empíricamente desarrollada", en el sentido de estar construido sobre las respuestas previas de los propios estudiantes, y no las ideas del investigador, como suele ser habitual en otros instrumentos (Aikenhead, 1988). Con ello se evita la doctrina de la percepción inmaculada, es decir, la hipótesis implícita en todos los cuestionarios que los respondientes y el investigador entienden y perciben el significado y el texto de las cuestiones de la misma exacta manera, lo cual evita la ambivalencia y distorsión de los significados en las respuestas. Los procesos metodológicos seguidos en el desarrollo empírico de las cuestiones dotan a las cuestiones de una validez inherente (Aikenhead y Ryan, 1992).

\section{Procedimiento}

Los cuestionarios han sido aplicados colectivamente, en los grupos clase de los alumnos que forman la muestra. La aplicación de los seis tipos distintos de cuestionarios se ha balanceado en cada grupo, entre hombres y mujeres, mediante una distribución aleatoria de cada tipo de cuestionario, estratificada por subgrupos de género. La respuesta en cada cuestión consiste en seleccionar la opción que se ajusta a la actitud más acorde con el pensamiento del que responde.

La literatura informa que las actitudes relacionadas con los temas científicos dependen de diversas variables: la cantidad de educación científica recibida por cada persona y el género son algunas de las más usuales (Vázquez y Manassero, 1995, 1996). La cantidad de educación científica recibida se ha operacionalizado en una variable denominada grado de exposición a la ciencia, a través de un sistema de puntuación proporcional a la cantidad de materias científicas cursadas por cada persona a lo largo de su currículo académico hasta el momento de responder el cuestionario, puntuación que depende del nivel, del curso y de la especialidad de los estudios seguidos por cada participante. Con esta operacionalización, los licenciados y estudiantes del último curso de ingenierías, físicas o químicas alcanzan las pun- 
tuaciones más altas, mientras los estudiantes de secundaria obligatoria, que todavía no han realizado la opción entre materias científicas y no científicas tienen la puntuación mínima. En base a estas puntuaciones la muestra total se ha dividido en tres categorías de exposición a la ciencia, baja, media y alta. Además, para el profesorado, se han considerado variables de edad y nivel educativo. Los resultados más relevantes obtenidos en cada uno de estos grupos se comentan brevemente, aunque no se ofrecen pormenorizados, debido a las limitaciones de espacio.

\section{Muestra}

La compleja situación de la diversidad de estudios y modalidades en las enseñanzas medias, debido al proceso de implantación anual de los estudios de reforma, provocaban cambios significativos anuales impredecibles de las poblaciones que impedían ningún diseño muestral previo. Las directrices aplicadas en la selección de la muestra han buscado la representatividad de cada uno de los niveles de enseñanza; la selección de participantes se hace por grupos-clase (muestreo por cuotas), para facilitar la aplicación de los cuestionarios; la selección de grupos se hizo al azar entre todos los niveles existentes en ese momento y aproximadamente proporcional a la población de cada estrato, ante la inexistencia de datos estadísticos previos seguros (muestreo estratificado con afijación aproximadamente proporcional).

La muestra de alumnado está compuesta por 4,132 estudiantes de todos los niveles y modalidades del sistema educativo (titulados, universitarios, y secundarios), existentes en el momento de la aplicación de los cuestionarios (años 1995 y 1996), en la isla de Mallorca, cuyas edades son superiores a los 14 años y el $95 \%$ de la muestra está en edades entre 14 y 27 años. La muestra en cada uno de los seis cuestionarios no es exactamente igual, debido a la mortalidad experimental (cuestionarios deficientemente contestados o incompletos), y se sitúa en torno a las 700 personas.

La muestra de profesorado está formada por 654 profesores (318 y 336 contestaron cada uno de los dos cuestionarios) de primaria $(46 \%)$, secundaria $(44 \%)$ y universidad $(10 \%)$.

\section{Resultados}

Como se ha explicado anteriormente los diversos temas planteados han sido respondidos por una muestra de alumnado y otra de profe- 
sorado, de todos los niveles educativos, especialidades y edades. Expondremos los resultados de estas respuestas separadamente para el alumnado (en primer lugar) y el profesorado (en segundo lugar), junto con el epígrafe de la cuestión a la que se refieren.

\section{Influencia general (alumnado)}

Las dos primeras cuestiones plantean la creencia sobre la influencia global de la sociedad sobre ciencia y tecnología y las razones que pueden fundamentar esta creencia.

La influencia de la sociedad sobre la ciencia es justificada mayoritariamente por el alumnado (más de un tercio) por la influencia general que la sociedad ejerce sobre todos sus miembros, en particular, también sobre los científicos (C, 38\%); los intereses de la sociedad se comunican de una forma natural a los científicos. Otras razones relevantes de la influencia social sobre la ciencia son la demanda social de conocimiento científico (B, $13 \%$ ), la subvención social de la ciencia (F, $12 \%$ ) y la proyección del conocimiento científico sobre los desarrollos tecnológicos $(\mathrm{E}, 12 \%)$.

20821 ¿La sociedad influye en la ciencia?

A. La sociedad no influye en gran medida sobre la ciencia

B. La demanda de la sociedad para comprender el mundo estimula la acumulación de conocimiento cientifico.

C. Los cientificos son miembros de la sociedad Cuando se extiende el interés de la sociedad por un terns, los cientificos están más inclinados a estudiar ese terna

D La sociodad concreta qué tipo de investigacion cientifica es aceptable basada en nuestros valores, morales y éticos

E La sociedad usa el conocimiento cientifico comio una base para el desarrollo de la tecnologia

F La sociedad influye sobre la ciencia a través de la subvención economica de la cual depende la mayoria de la investigación

G I sociedad acepta o rechaza la tecnologia, creando mayor o menor demanda por la ciencia

1 No entiendo la cuestión

2 No se lo suficiente sobre el terna para seleccionar una opcion

3 Ninguna de las opciones satisface bésicamente mı opinion

Alumnado $\mathrm{N}=655$ Casos validos 654

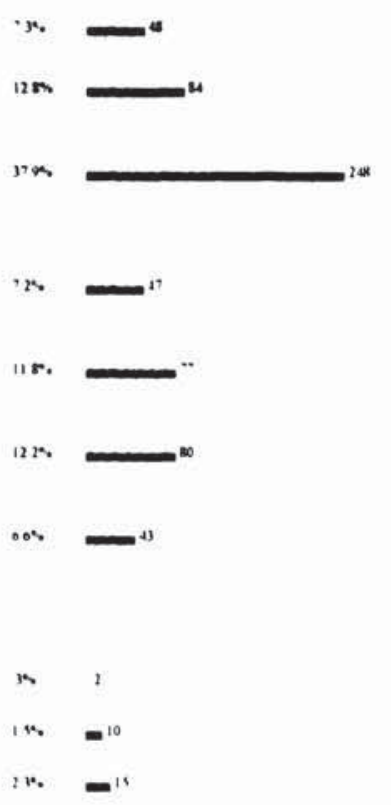


Las diferencias de actitudes según el grupo de exposición a la ciencia no son relevantes y tampoco las diferencias de género cuando se controla el grupo de exposición a la ciencia. En este último caso, se puede notar simplemente que las mujeres de alta exposición a la ciencia eligen en mayor proporción que los hombres la opción B (demanda social de conocimiento científico), y en menor proporción la mayoritaria opción C.

La influencia de la sociedad sobre la tecnología es justificada mayoritariamente en base a las demandas de la sociedad (B, 27\%), sin más, o atemperadas éstas por los valores propios de la sociedad que limitan las mejoras (F, 27\%), entre ambas opciones más de la mitad de la muestra. El resto de las alternativas tienen proporciones menores; entre ellas, otras formas de reconocer la influencia de la sociedad sobre la tecnología con una proporción relevante de respuestas son a través de la ciencia (considerando implícitamente la tecnología como ciencia aplicada), y a través de medios legales y políticos que regulan y limitan el uso de la tecnología.

$20811_{¿} L a$ sociedad influye en la tecnología?

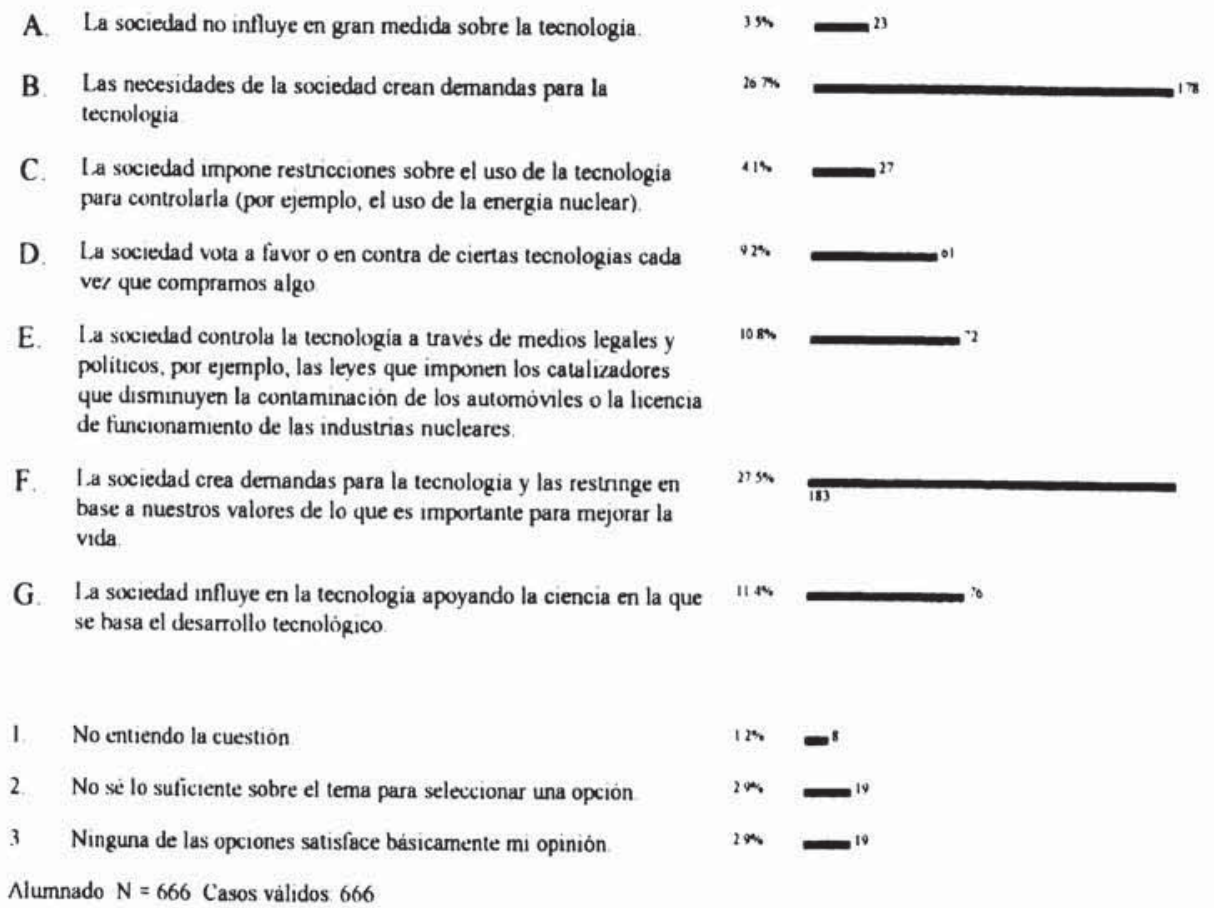


Las diferencias de actitudes según el grupo de exposición a la ciencia muestran que el grupo de exposición a la ciencia media elige más que los otros dos grupos la genérica opción $\mathrm{B}$, mientras que el grupo de exposición a la ciencia alta elige más la opción F; el grupo de baja exposición a la ciencia elige las opciones mayoritarias menos que los otros dos grupos. Resulta llamativo que el grupo de estudiantes de alta exposición a la ciencia tienen la tasa más alta de elección de la opción $\mathrm{F}$, la que tiene en cuenta los valores sociales como un elemento restrictivo respecto a la tecnología. Las diferencias de género importantes se centran también en esta opción $\mathrm{F}$ : las mujeres de exposición a la ciencia baja y media eligen esta opción en mayor proporción que los hombres; las mujeres de alta exposición a la ciencia eligen la opción $\mathrm{B}$ en menor proporción que los hombres.

\section{Gobierno (alumnado)}

El deber del gobierno de subvencionar la investigación científica se justifica mayoritariamente por el alumnado porque comprendiendo mejor nuestro mundo, los científicos pueden convertirlo en un lugar mejor para vivir (D, casi la mitad de respuestas), y en menor proporción, que sólo debe subvencionarse la investigación referida, exclusivamente, a la salud, el medio ambiente o la agricultura (E, 18\%). En minoría quedan los que justifican la investigación para evitar la dependencia del país de otros (A), satisfacer la curiosidad de conocer (B) o, incluso, a sabiendas del riesgo de no poder conocer de antemano los beneficios alcanzables (C). Prácticamente nadie (F, 0.6\%) opina que no debería gastarse dinero en investigación científica, para dedicarlo a ayudas sociales directas a los más necesitados.

Las diferencias de las actitudes de los estudiantes según el grupo de exposición a la ciencia se centran en las opciones minoritarias B y C: el grupo de alta exposición a la ciencia elige estas razones en mayor proporción que los otros dos grupos. El grupo de baja exposición a la ciencia tiene tendencia a seleccionar menos que los otros dos grupos la opción mayoritaria (D).

\section{Gobierno (profesorado)}

El deber del gobierno de subvencionar la investigación científica se justifica abrumadoramente por el profesorado (dos tercios) con el 
M. ${ }^{a}$ Antonia Manassero Mas y Ángel Vázquez Alonso

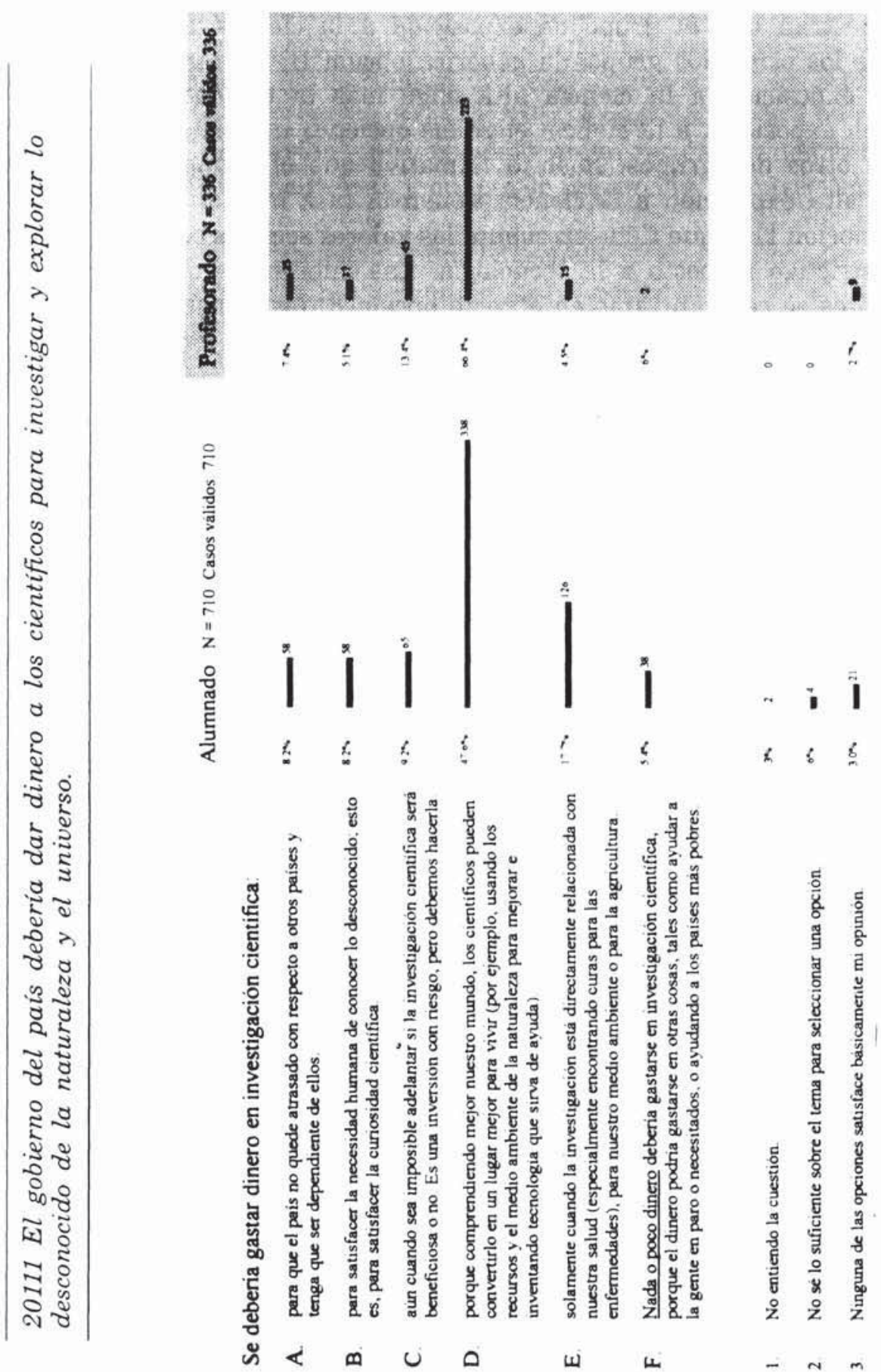


argumento de la mejora de la calidad de vida (comprendiendo mejor nuestro mundo, los científicos pueden convertirlo en un lugar mejor para vivir). El resto de los argumentos quedan reducidos a una proporción baja, aunque entre ellos es interesante resaltar el que se refiere a asumir el riesgo de no poder conocer de antemano los beneficios alcanzables de la investigación (C), que parece más propio de personas adultas, y la principal diferencia respecto al alumnado, por su importancia relativa. Sólo dos profesores opinan que no debería gastarse dinero en investigación científica, para dedicarlo a ayudas sociales directas a los más necesitados.

Las diferencias de actitudes del profesorado según el grupo de exposición a la ciencia son difíciles de establecer más allá de simples tendencias debido a la descompensación de las muestras en cada grupo. La tendencia observable más relevante es que los profesores de alta exposición a la ciencia apoyan menos la opción mayoritaria D y relativamente más que los otros dos grupos la opción de asumir el riesgo de desconocer los beneficios posibles de la investigación.

Industria (alumnado)

La posibilidad de que un control de la ciencia por las empresas fuera mejor para la ciencia es ampliamente rechazada por el alumnado (dos tercios en contra). Las dos razones principales seleccionadas son considerar que los descubrimientos estarían limitados a aquellos orientados a producir ganancias a la empresa y no tanto por los beneficios de interés general (D, $32 \%)$, y porque los descubrimientos de la ciencia no pueden ser controlados por las empresas, ni siquiera por los propios científicos $(\mathrm{F}, 27 \%)$. Los partidarios del control de la ciencia por las empresas (un cuarto) justifican su actitud por considerar que la competitividad y mejor dotación de las empresas hará descubrimientos más rápidos y útiles $(\mathrm{A}, 13 \%)$ y porque mejorará la cooperación entre ciencia y tecnología $(\mathrm{B}, 8 \%)$.

\section{Industria (profesorado)}

El control de la ciencia por las empresas tiene un perfil de respuesta entre el profesorado prácticamente idéntico al del alumnado. El profesorado también rechaza abrumadoramente el control de la ciencia por las empresas (dos tercios), principalmente por considerar que los descubrimientos estarían orientados a producir ganancias a la empresa (D, $31 \%$ ) y porque ni siquiera las empresas pueden controlar lo que la ciencia descubrirá $(\mathrm{F}, 28 \%)$. La diferencia respecto al alumnado se 
M. ${ }^{a}$ Antonia Manassero Mas y Ángel Vázquez Alonso

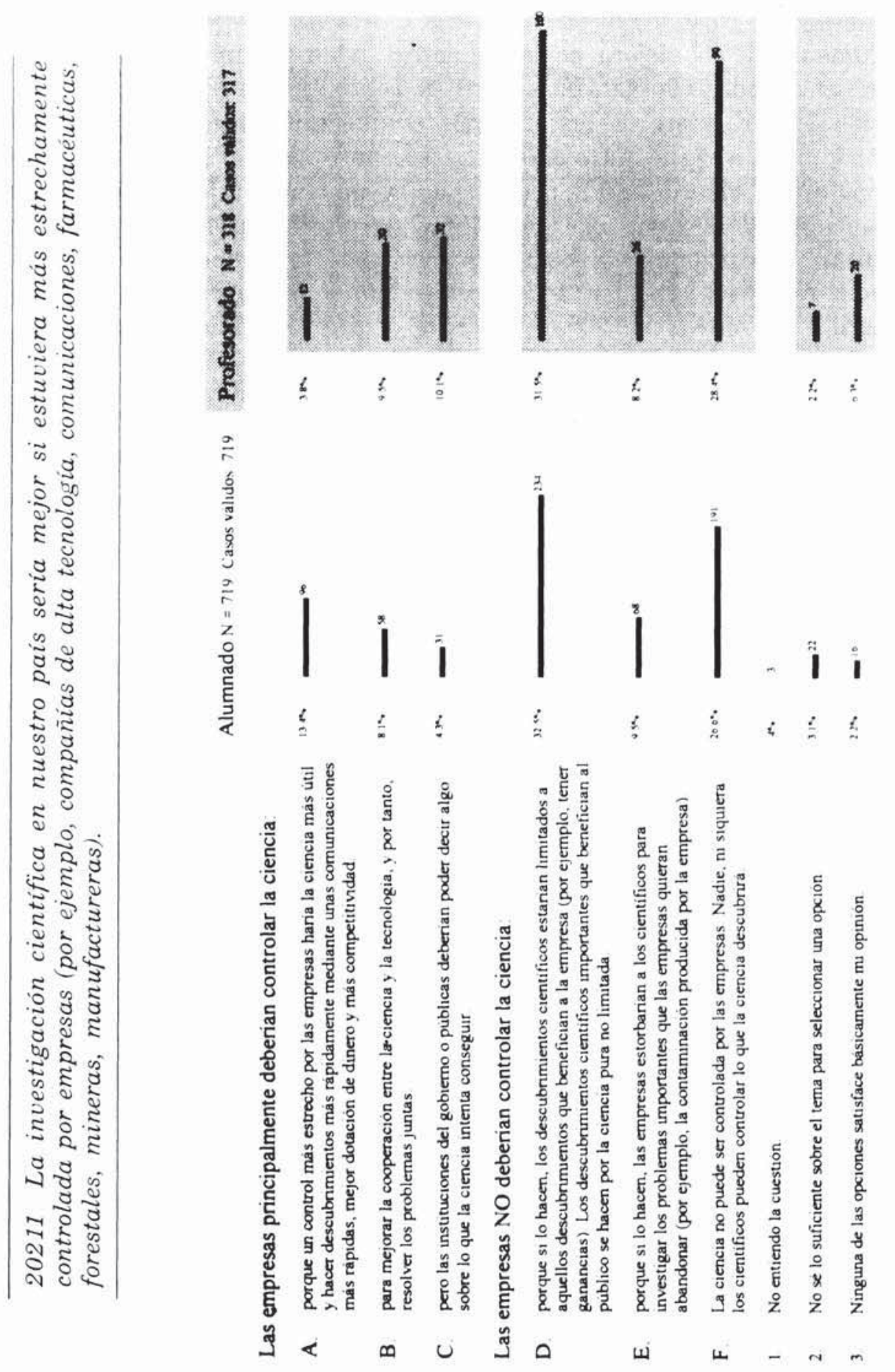


produce en el perfil de los partidarios del control de la ciencia por las empresas (un cuarto), que justifican su actitud por considerar que las instituciones públicas tienen que participar en este proceso (C, $10 \%$ ) y porque la empresa puede mejorar la cooperación entre ciencia y tecnología (B, 9\%).

Las diferencias de actitudes según el grupo de exposición a la ciencia o el género, tanto entre el alumnado como entre el profesorado, sobre el control de la ciencia por las empresas no son relevantes. $\mathrm{Si}$ acaso, cabe notar como tendencia global, que los estudiantes de alta exposición a la ciencia son menos favorables que los otros grupos respecto al control de la ciencia por empresas.

\section{Investigación militar e industrial (alumnado)}

La comparación entre investigación con fines militares/industriales e investigación con fines sociales (salud, agricultura, o la simple curiosidad) muestra que el alumnado cree mayoritariamente que la mayor parte de la investigación es militar/industrial (menos de la mitad). Sin embargo, la opción singular mayoritariamente seleccionada (un cuarto) es ecléctica, en cuanto que considera que la investigación militar es dominante sólo en algunos países muy empeñados en sus gastos de defensa, mientras que en otros domina la investigación social (D, $24 \%$ ). La segunda opción en importancia justifica la investigación militar e industrial porque viene condicionada por el dinero, y no tanto por la curiosidad o el placer de saber (C, 18\%).

Las diferencias de actitudes según el grupo de exposición a la ciencia entre el alumnado muestra la tendencia del grupo de alta exposición a la ciencia más favorable a justificar la mayor importancia de la investigación militar e industrial por el dinero dedicado a ella (C). En diferencias de género, las mujeres estudiantes de alta exposición a la ciencia apoyan más la ecléctica opción que se investiga mucho en todas las áreas (E), mientras apoyan menos la razón del dinero (C).

\section{Investigación militar e industrial (profesorado)}

La comparación entre investigación militar/industrial e investigación social muestra una opinión del profesorado más abrumadora en favor del dominio de la investigación militar e industrial (dos tercios). Como 
M. "Antonia Manassero Mas y Ángel Vázquez Alonso
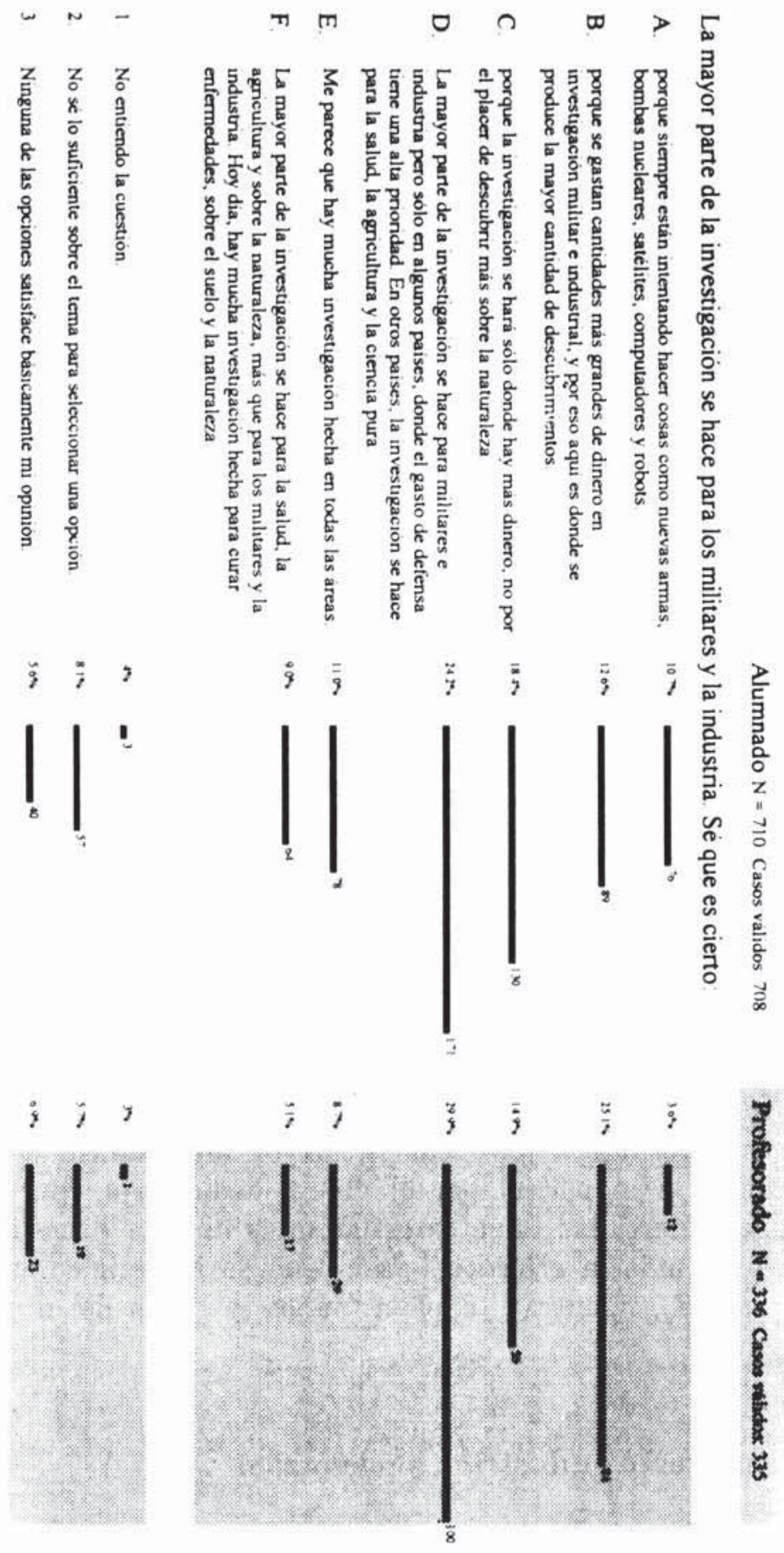

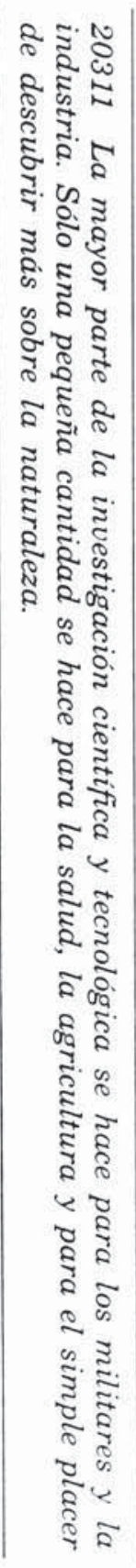


en el caso del alumnado, la opción singular dominante es la posición ecléctica, que afirma que sólo en algunos países domina la investigación militar (D, 30\%). La principal diferencia respecto a la opinión del alumnado es la segunda opción mayoritaria elegida por el profesorado, reconociendo que las elevadas cantidades de dinero dedicadas a la investigación militar/industrial son las que determinan la mayor cantidad de descubrimientos en estas áreas (B, 25\%).

\section{Creencias éticas y religiosas (alumnado)}

El alumnado cree que las creencias éticas y religiosas propias de una cultura influyen sobre la investigación científica, pues casi dos tercios $(59 \%)$ de los encuestados responden con opciones en esta línea. La razón más invocada alude a la forma personal que cada uno tenemos de tener en cuenta nuestra propia identidad cultural (23\%). La segunda opción más frecuente sostiene el punto de vista opuesto (las creencias no influyen en la investigación), basada en la tesis de la independencia entre la actividad científica y las creencias personales (19\%). Otras dos razones relevantes seleccionadas, de uno y otro signo, se refieren al interés de determinados grupos culturales y religiosos para promocionar o no promocionar determinadas investigaciones y a la creencia que ni los choques ni controversias con los grupos religiosos o culturales afectan a la investigación científica.

Globalmente, las mujeres tienen actitudes más desfavorables a la influencia de las creencias éticas y religiosas sobre la investigación, que sus homólogos hombres.

\section{Instituciones educativas (alumnado)}

La importancia estratégica de la ciencia y la tecnología para el desarrollo y prosperidad de un país requiere científicos, técnicos e ingenieros, y por tanto, es necesario que exista una cultura general del público sobre la ciencia y la tecnología. Más de un tercio de los estudiantes creen que cuanto más se aprenda sobre ciencia y tecnología, el público estará mejor informado, tendrá mejores opiniones y aportará mejores contribuciones sobre el uso de ciencia y tecnología. Otra quinta parte del alumnado cree que aprender sobre ciencia y tecnología permitirá al público tener una mejor imagen de ciencia y tecnología, entender mejor las opiniones de los expertos y apoyarlas. En sentido 

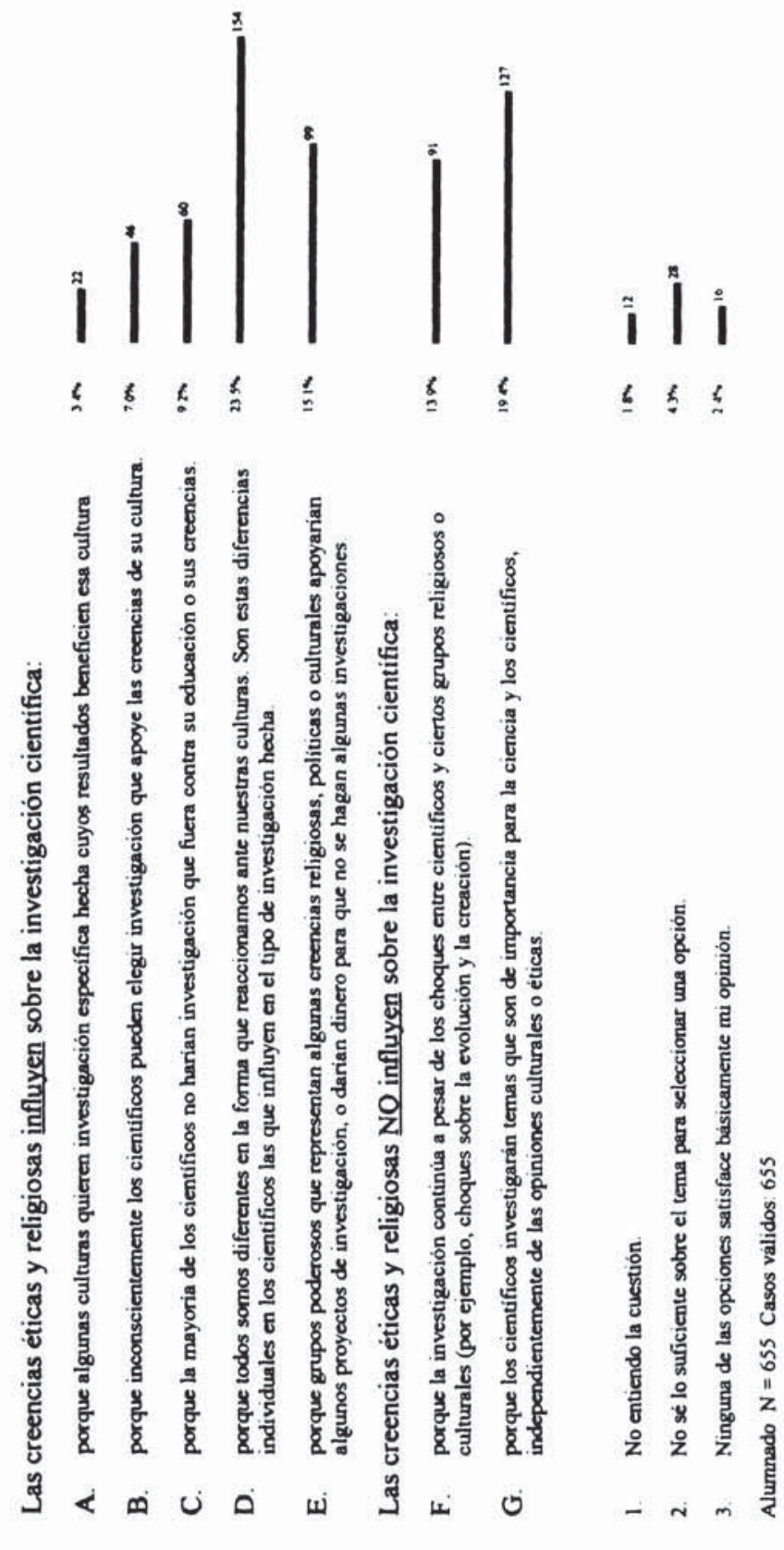
Actitudes hacia la influencia de la sociedad...

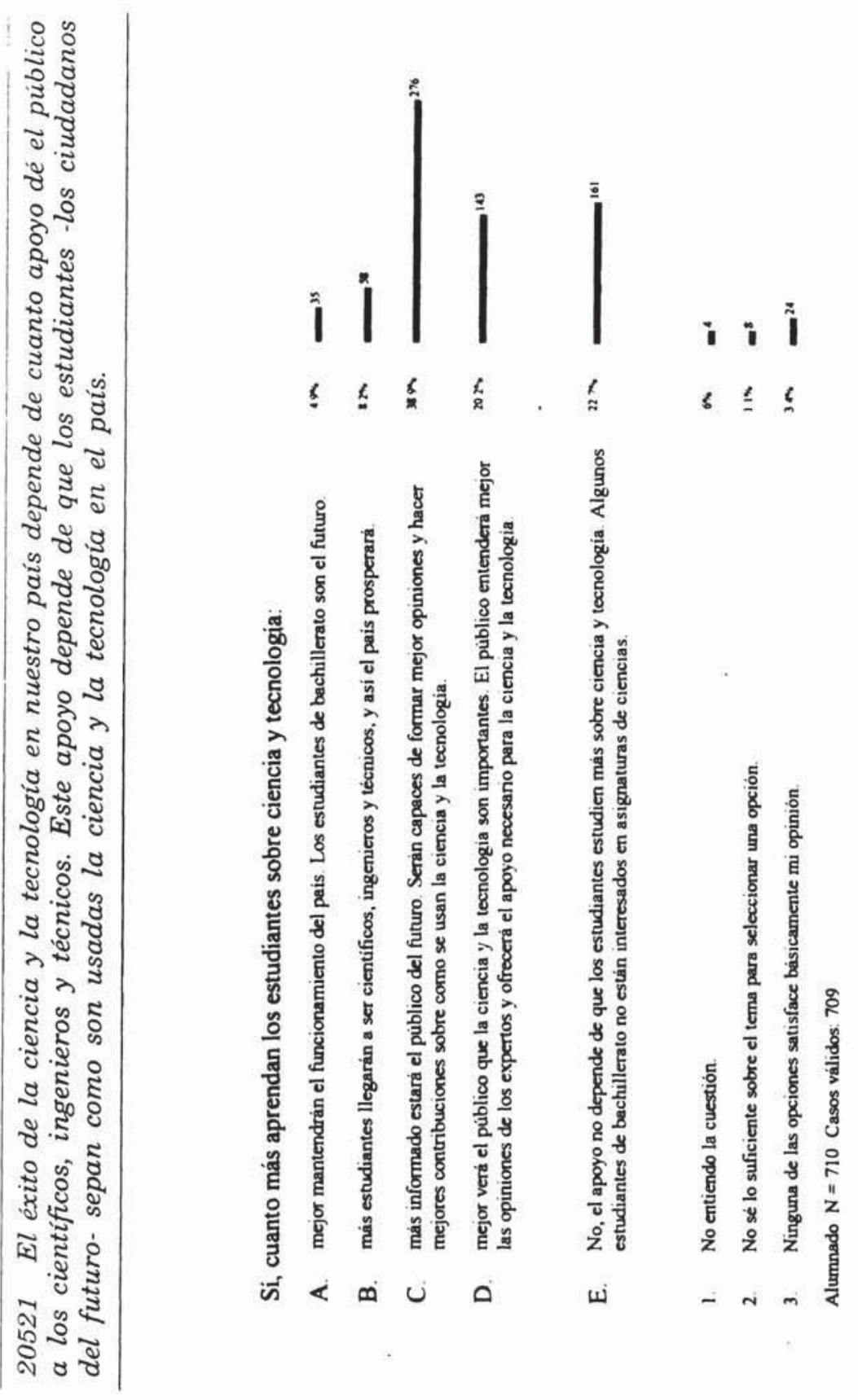


contrario, otra quinta parte del alumnado no cree que aprender más sobre ciencia y tecnología redunde en un éxito de apoyo para la ciencia y tecnología, porque mucha gente no tiene ningún tipo de interés en ciencia y tecnología.

El grupo de alta exposición a la ciencia es más partidario de la opción mayoritaria (C) que el grupo de exposición a la ciencia media y éste, a su vez, que el grupo de exposición a la ciencia baja. El patrón compensador inverso se observa en el caso de la opción E (no importa que los estudiantes aprendan más ciencia y tecnología), apoyada en mayor medida por el grupo de baja exposición a la ciencia.

\section{Grupos de especial interés (alumnado)}

El desarrollo científico y tecnológico ha penetrado profundamente en las sociedades desarrolladas y, en ocasiones, ha producido efectos negativos desastrosos que impactan en la sensibilidad de la gente. Esta sensibilización social ha creado grupos de personas comprometidas en el control de determinadas decisiones científicas y tecnológicas (ecologistas, organizaciones religiosas, por derechos de los animales, etc.). La influencia sobre la ciencia y tecnología de estos grupos de especial interés, que se pueden manifestar a favor o en contra de determinadas áreas de investigación científica, es reconocida mayoritariamente por casi la mitad del alumnado; la razón más importante alegada de esta actitud es la influencia de los grupos influencia en la opinión pública, y a su través, en los científicos (27\%). Paralelamente, una quinta parte del alumnado adopta una posición ecléctica, reconociendo la influencia de los grupos pero desmitificando su poder, porque estos grupos no siempre tienen éxito, ya que científicos y tecnólogos tienen la última palabra (F, 20\%). En conjunto, casi una cuarta parte del alumnado considera que los proyectos de ciencia y tecnología no son influidos por estos grupos; de éstos, la mayoría de ellos fundamenta la falta de influencia de estos grupos porque el gobierno y los técnicos no se dejan influir por ningún grupo, cuando ya han decidido realizar un proyecto $(\mathrm{H}, 20 \%)$.

Las diferencias de actitudes según el grupo de exposición a la ciencia muestran que el grupo de baja exposición a la ciencia apoya menos la influencia de los grupos de especial interés en la opinión pública (opción mayoritaria $\mathrm{C}$ ) y el grupo de alta exposición a la ciencia apoya menos que los otros dos la opción ecléctica $(\mathrm{F})$ que relativiza el poder de los grupos. Esta misma opción es más apoyada por las mujeres 
Actitudes hacia la influencia de la sociedad...

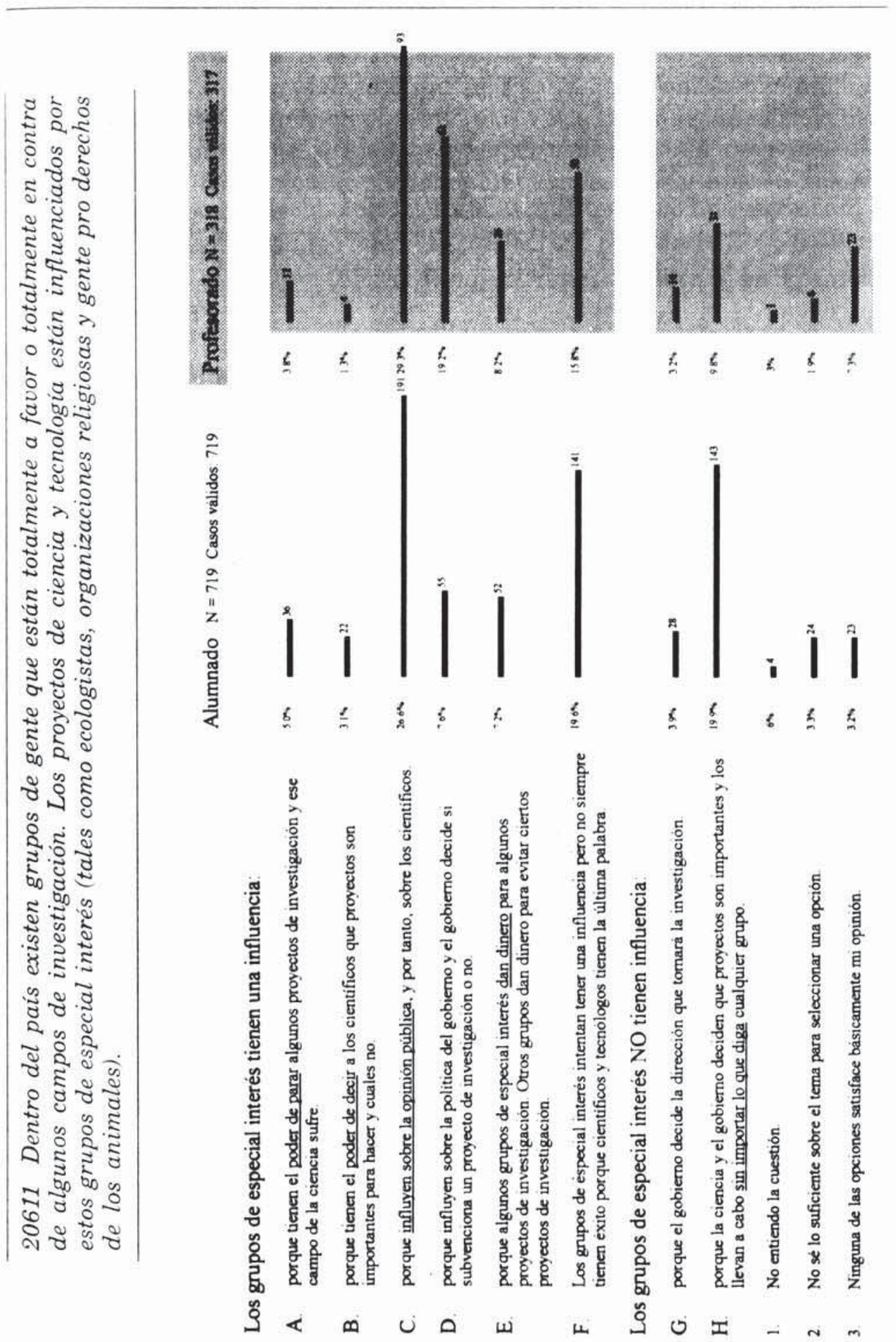


de baja exposición a la ciencia; las mujeres del grupo de alta exposición a la ciencia apoyan más que los hombres la opción mayoritaria, mientras éstos apoyan más que las mujeres la ausencia de influencia de estos grupos porque las decisiones del gobierno no los tienen en cuenta $(\mathrm{H})$.

\section{Grupos de especial interés (profesorado)}

La actitud del profesorado respecto a la influencia de los grupos de especial interés sobre la ciencia y tecnología tiene un perfil similar al del alumnado, pero cuantitativamente muestra algunas diferencias. Esta influencia es considerada importante por más de la mitad del profesorado y la razón más importante es su influencia en la opinión pública, y a su través, en los científicos; la segunda razón, muy importante también, es su influencia sobre el gobierno y su política de subvenciones. Menos de una quinta parte del profesorado se adhiere a la posición ecléctica que desmitifica el poder de estos grupos porque no siempre tienen éxito, ya que científicos y tecnólogos tiene la última palabra. El grupo de profesorado que no cree en la influencia de estos grupos es la mitad que entre el alumnado, y atribuyen su creencia principalmente a que el gobierno y los técnicos no se dejan influir por ningún grupo, cuando ya han decidido realizar un proyecto. En conjunto, el profesorado tiene una actitud más favorable que el alumnado respecto a la influencia social de los grupos especiales, de modo que aunque las razones mayoritarias a favor y en contra son casi las mismas, aquellas tienen tasas más altas entre el profesorado que las contrarias. El profesorado atribuye la influencia de los grupos significativamente más que el alumnado a su influencia sobre el gobierno, debido a la capacidad de éste para financiar proyectos de investigación.

\section{Discusión}

Las relaciones entre la sociedad y la ciencia han sido excluidas deliberadamente de la epistemología de la ciencia por el pensamiento positivista, con la pretensión de fundamentar una visión de la ciencia, neutral, objetiva e impermeable a influencias externas. En este marco, el valor absoluto de verdad del conocimiento científico y su progreso acumulativo resulta más fácil de establecer y defender recurriendo a unos hechos científicos incontrovertibles e incontaminados. Desafortunadamente, los análisis históricos y sociológicos han echado por tierra 
esta visión, mostrando las intrincadas y continuas relaciones entre la sociedad y la ciencia, como por otra parte, parecería de sentido común admitir, o al menos, suponer. Puesto que el pensamiento positivista ha tenido una influencia penetrante, especialmente en los ambientes científicos y técnicos, donde se han formado la mayoría de los profesores, por ejemplo, plantear la cuestión de la influencia de la sociedad en la ciencia y tecnología no sólo es un test al pensamiento positivista sino a las actitudes generales sobre la ciencia y tecnología en relación con la sociedad.

Globalmente, una mayoría de estudiantes admiten la influencia de la sociedad sobre la ciencia y tecnología, como se puede concluir de los resultados en las diferentes cuestiones evaluadas en este estudio. Este resultado es especialmente patente en las dos cuestiones iniciales que plantean genéricamente la influencia social sobre ciencia y tecnología (20711 y 20811); las justificaciones basadas en causas más generales son apoyadas con mayor intensidad que las razones más concretas, pero la influencia de la sociedad sobre ciencia y tecnología se reconoce con abrumadora unanimidad, acumulando unas u otras razones.

Cuando la influencia de la sociedad se concreta en aspectos particulares, tales como la influencia de la cultura religiosa o ética o los grupos de especial interés, la tasa de quienes tienen una actitud favorable a la influencia de la sociedad sobre ciencia y tecnología disminuye (más de. la mitad de la muestra en el primer caso y un quinto en el segundo).

La influencia social sobre la ciencia y tecnología vehiculada a través de factores concretos de tales como el gobierno, las empresas industriales, el ejército o la educación científica y tecnológica del público muestran actitudes de diferente intensidad. Apenas un $5 \%$ de estudiantes y sólo dos profesores creen que el gobierno no debe invertir dinero en investigación; en torno a una cuarta parte de estudiantes y profesorado son partidarios del control de la investigación por las empresas; aproximadamente el $40 \%$ de estudiantes y profesorado creen que la mayor parte de la investigación se hace para los militares y la industria, y el $23 \%$ de los estudiantes opinan que el apoyo del público a la ciencia no depende de estudiar más ciencia y tecnología.

El modelo de respuesta única aplicado a las cuestiones no permite obtener una puntuación global sobre las cuestiones estudiadas, a modo de valoración ponderada total de los diversos aspectos representados en ellas. En consecuencia, la aproximación discursiva e independiente a cada una de las cuestiones es la única síntesis posible en estas 
condiciones. En conjunto, los resultados obtenidos sugieren una actitud global de los jóvenes estudiantes encuestados, desde secundaria a la universidad, muy favorable a la influencia de la sociedad sobre el sistema de ciencia y tecnología.

Dadas las limitaciones metodológicas citadas del modelo de respuesta única empleado, un análisis comparativo entre grupos puede aportar una referencia interesante para alcanzar una interpretación más profunda de los resultados alcanzados. En el estudio se ha definido una variable denominada exposición a la ciéncia que mide la cantidad de educación científica recibida por las personas encuestadas y el género. Como grupos de referencia para la comparación se pueden utilizar los resultados del profesorado y los resultados de otros estudios con las mismas cuestiones (Aikenhead, Ryan, 1989; Rubba y Harkness, 1993).

La variable exposición a la ciencia permitiría contrastar la hipótesis sobre la impregnación de la filosofía positivista de la educación en ciencias. Las cuestiones generales sobre la influencia social en ciencia y tecnología no muestran diferencias importantes, y en el único caso en que éstas se observan (influencia social sobre la tecnología), el grupo de estudiantes de alta exposición a la ciencia tienen la tasa más alta en la opción que sugiere que los valores sociales personalizan las demandas sociales respecto a la tecnología. Por tanto, los estudiantes con mayor educación científica, indirectamente, no sólo valoran la influencia social, sino que valoran relativamente más el protagonismo de los valores particulares de cada sociedad, un rasgo negado repetidamente por el positivismo.

Sin embargo, en la financiación de la ciencia y tecnología por el gobierno, el grupo de alta exposición a la ciencia se diferencia de los otros grupos en justificarla más por la necesidad de conocer y el carácter de riesgo imprevisto que siempre tiene la investigación, dos razones que podrían entroncar más directamente con el pensamiento epistemológico general. Támbién este grupo justifica más que los demás la importancia de la investigación militar e industrial por la mayor presencia de dinero en estos ámbitos, una visión poco idealizada y muy realista.

En el caso de la educación científica de los ciudadanos, como sería plausible esperar, el grupo de alta exposición a la ciencia apoya más intensamente la opción mayoritaria en favor de una mayor información y educación del público. En el caso de la influencia de los grupos de especial interés, el grupo de alta exposición a la ciencia tiene una actitud más favorable que los otros a el poder influyente de estos grupos, especialmente por su incidencia en la opinión pública. 
En suma, una alta exposición a la ciencia no parece determinar diferencias profundas en las actitudes del alumnado respecto a la influencia social sobre la ciencia y tecnología y mucho menos evidenciar tendencias positivistas en este grupo. Algunas cuestiones no presentan diferencias (influencia global sobre la ciencia, control de la ciencia por las empresas, influencia de la cultura) y las diferencias observadas en las demás cuestiones están muy localizadas en unas pocas opciones de respuestas. Las diferencias observadas en el grupo de alta exposición a la ciencia no inducen a pensar que este grupo mantenga actitudes más próximas o concomitantes con una filosofía positivista presuntamente trasmitida en su educación, antes al contrario, alguno de los rasgos encontrados (importancia de los valores sociales en la determinación de la tecnología) sugieren justamente el patrón contrario. Para concluir, la exposición a la ciencia no sólo no determina diferencias profundas en la visión de la influencia de la sociedad sobre ciencia y tecnología entre los estudiantes, sino que las pocas diferencias encontradas no sugieren tampoco una actitud positivista.

La comparación con la muestra de estudiantes canadienses del estudio original (Aikenhead, Ryan, 1989) permite contrastar todas las cuestiones. Sin embargo, debe tenerse en cuenta que la muestra canadiense corresponde a estudiantes de bachillerato, no es estrictamente equivalente a nuestra muestra, que incluye también estudiantes universitarios.

El perfil de respuestas de los estudiantes canadienses sobre la financiación de ciencia y tecnología por el gobierno es prácticamente idéntico al de los estudiantes españoles.

El perfil de respuestas de los estudiantes canadienses sobre el control de la ciencia y tecnología por las empresas difiere ligeramente de los estudiantes españoles; éstos muestran un proporción más alta $(27 \%)$ que aquéllos (12\%) sobre la idea que la ciencia no puede ser controlada por nadie ya que nadie puede controlar lo que la ciencia descubrirá. La actitud de los estudiantes españoles es más contraria al control de la ciencia por las empresas, gozando de un mayor apoyo la idea que este control no es posible.

Los estudiantes canadienses creen que la mayor parte de la investigación se realiza para los militares y la industria (en Norteamérica) en mayor proporción que los estudiantes españoles.

La influencia social de la cultura religiosa y ética sobre la ciencia es más admitida por los estudiantes españoles (59\%) que los canadienses (44\%); sin embargo, las razones principales en los grupos favorables y desfavorables a esta influencia tienen un perfil bastante paralelo 
en una y otra muestra, aunque con las naturales diferencias cuantitativas debido a las diferencias en las proporciones citadas.

La necesidad de aprender más sobre ciencia y tecnología para estar más preparados como ciudadanos, es bastante apoyada por los estudiantes y con proporciones no muy diferentes; sin embargo, la proporción de españoles desfavorables a apoyar esta relación es más amplia (23\%) que entre los canadienses (7\%). Este rasgo podría ser un indicador de un menor interés en estudiar ciencia y tecnología entre los estudiantes españoles.

El perfil de la cuestión referida a la influencia de grupos de especial interés (ecologistas, etc.) sobre ciencia y tecnología difiere bastante entre canadienses y españoles. Aunque ambos grupos se muestran mayoritariamente a favor de la influencia de estos grupos, la proporción es significativamente diferente: los españoles (24\%) apoyan la no influencia de estos grupos más que los canadienses (12\%). Las diferencias más relevantes en las alternativas seleccionadas por los grupos favorable y desfavorable a esta influencia aparecen en la influencia sobre la política del gobierno, más frecuente entre los canadienses, mientras que los españoles se decantan más por la posición ecléctica (influyen pero no tienen mucho éxito) y la desfavorable a la influencia de los grupos (al gobierno y a los científicos no les importan estos grupos).

En suma, la comparación con los estudiantes canadienses no ofrece diferencias relevantes en las actitudes sobre la influencia de la sociedad sobre la ciencia y tecnología. Las diferencias locales observadas podrían ser debidas a las diferencias sociales y culturales entre Canadá y España. Así los canadienses son más favorables al control de ciencia y tecnología por las empresas, al protagonismo de militares e industria en la cantidad de investigación y a una mayor influencia de los grupos de especial interés; los españoles apoyan más la influencia de los factores culturales religiosos y éticos. Dos rasgos actitudinales podrían sugerir una menor sensibilidad hacia la ciencia y tecnología de los estudiantes españoles: por un lado, la mayor increencia en la necesidad de estudiar más ciencia y tecnología, y por otro, la tendencia un poco mítica de ver la ciencia y tecnología como algo cuyos descubrimientos no se pueden controlar. No obstante, la falta de equivalencia estricta de la muestras comparadas no permite una atribución precisa de las diferencias observadas.

Las respuestas del grupo de profesores constituyen otra referencia adicional a la hora de valorar los resultados obtenidos. Aunque se han identificado algunas diferencias entre las actitudes del alumnado y el profesorado en las cuestiones respondidas por ambas muestras 
(subvención del gobierno, control por las empresas, investigación militar e industrial e influencia de los grupos de especial interés), la valoración global, sin embargo, muestra una semejanza bastante pronunciada entre las actitudes de ambos grupos. La influencia de los grupos de especial interés es la única cuestión que podría marcar diferencias relevantes entre alumnado y profesorado, teniendo éstos una actitud más favorable a la influencia real de estos grupos por su incidencia en la política del gobierno, contrastando con la creencia de los estudiantes de que gobierno y científicos actúan sin tener en cuenta estos grupos. La semejanza de actitudes entre alumnado y profesorado no resulta sorprendente, sobre todo si se tiene en cuenta que el profesorado actúa impartiendo la educación escolar, donde se conforman muchas de las actitudes del alumnado; parece lógico que los educandos adquieran las actitudes de sus profesores, incluso las actitudes no manifiestas, especialmente cuando la educación de las actitudes no ha sido incluida en el currículo escolar habitual. La mayor madurez del profesorado (por edad y formación) podría justificar las pequeñas diferencias observadas (mayor importancia de los grupos de especial interés o de la investigación militar e industrial atribuida a la disposición de mayores subvenciones). En suma, se podría decir que la muestra de estudiantes jóvenes tiene las mismas actitudes hacia la ciencia y tecnología que la muestra adulta de profesorado; se podría interpretar que el grueso de la actitudes hacia la influencia social sobre ciencia y tecnología se conforma en la juventud y cambian poco en la madurez. Esta conclusión es un factor en favor de una mayor y mejor educación de las actitudes relacionadas con la ciencia y tecnología en la escuela (Vázquez y $\mathrm{Ma}$ nassero, 1995).

Como se ha dicho, la actitud global de los estudiantes es mayormente favorable a la influencia de la sociedad sobre la ciencia. Sin embargo, cuando se analizan las justificaciones o razones mayoritarias de esa actitud global expresada a través de las elecciones, desde la perspectiva de la coherencia de estas razones con los conocimientos de historia, epistemología y sociología de la ciencia, las actitudes muestran rasgos de inadecuación en algunas de las razones mayoritarias. Así, la mitificación de la ciencia como algo incontrolable (opción F, 20211), la ingenuidad de que la investigación militar e industrial es mayoritaria sólo en algunos países (D, 20311), la opinión tecnocrática que científicos y tecnólogos tienen la última palabra en las decisiones (F, 20611) y ciencia y gobierno toman decisiones sin importar las opiniones de otros (H, 20611). Profundizar más este análisis permitiría valorar con mayor precisión las actitudes sobre cada tema, pero requeriría tener una 
baremación detallada de todas las posiciones en las cuestiones, tema que estamos desarrollando actualmente.

Finalmente, el tema educativo de fondo en este trabajo se refiere a una visión comprensiva de la ciencia y tecnología en un contexto social, es decir, interaccionando con la sociedad. La enseñanza tradicional de la ciencia, inspirada por la epistemología positivista, entre otros rasgos, se caracteriza por presentar dogmáticamente los hechos, conceptos y principios de la ciencia como verdades incontrovertibles, presentar algorítmicamente la metodología científica, separando artificialmente teoría y práctica, y excluir los valores contextuales; la consecuencia de este enfoque es un currículo descontextualizado, dominado por la lógica de la ciencia y por los contenidos factuales, que ignora las experiencias y conocimientos previos y produce una formación abstracta, alejada de la vida diaria de los estudiantes. Para evitar estos defectos, el movimiento de renovación en la enseñanza de la ciencia denominado ciencia-tecnología-sociedad sugiere un enfoque contextualizado, basado en las relaciones mutuas entre ciencia, tecnología y sociedad, que promueve una visión global de los problemas, como complejos procesos de toma de decisiones, y adaptándose a la personalidad y experiencias sociales de los estudiantes. La reforma contextualizada - CTS de la enseñanza de la ciencia no es fácil, pues la enseñanza tradicional y simplista de la ciencia, a pesar de sus evidentes fracasos, se haya bien implantada en la percepción dominante del público y del profesorado (Koul y Dana, 1997); los cambios curriculares hacia la reforma son complejos, pues no sólo requieren la puesta a punto de materiales nuevos, sino la transformación global del sistema educativo (diseño curricular, evaluación y exámenes, formación del profesorado, etc.). En este proceso de cambio, los resultados obtenidos aquí constituyen un argumento más en favor de la reforma CTS, ya que se demuestra que las actitudes de alumnado y profesorado son sensibles a la influencia de la sociedad sobre la ciencia y tecnología, a pesar de no haber tenido una educación en ciencias contextualizada. Este resultado tiene un doble valor contradictorio: por un lado, se diagnostica que las actitudes e ideas previas sobre este tema serían acordes con una concepción contextual de ciencia y tecnología, de modo que una educación CTS sería más respetuosa y coherente con las ideas previas del alumnado; por otro lado, a pesar de educar en contra de esta concepción previa, se constata que el sistema tradicional de enseñanza de la ciencia resulta tan aceptable en la opinión pública y en el pensamiento del profesorado. En esta contradicción asumida por el sistema radican gran parte de las dificultades de la reforma CTS para la educación en ciencia. 


\section{Bibliografía}

AIKENHEAD, G.S. y RYAN, A.G. (1989): The development of a multiple choice instrument for monitoring views on Science-Technology-Society topics. Final Report of SSHRCC Grant: Autor.

AIKENHEAD, G.S. y RYAN, A.G. (1992): The development of a new instrument: "Views on Science-Technology-Society" (VOSTS). Science Education, 76(5), 477-492.

Aikenhead, G.S., RYAN, A.G. y Fleming, R.G. (1989): Views on Science-Technology-Society Form CDN.MC.5. Department of Curriculum Studies College of Education, Autor.

ATIENZA, J. y LUJÁN, J.L. (1997): La imagen social de las nuevas tecnologías biológicas en España. Centro de Investigaciones Sociológicas, Madrid.

BERnal, J.D. (1979a): Historia social de la ciencia. Vol. 1 La ciencia en la historia. Península, Barcelona.

BERNAL, J.D. (1979b): Historia social de la ciencia. Vol. 2 La ciencia en nuestro tiempo. Península, Barcelona.

CIRES (Centro de Investigaciones sobre la Realidad Social). (1992): Actitudes sociales hacia la ciencia y la tecnología. (CIRES 10). Autor, Madrid.

DURANT, J.R., Evans, G.A. y Thomas, G.P. (1989): The public understanding of science. Nature, 340, 11-14.

Knorr-Cetina, K. (1981): The manufacture of knowledge. Pergamon Press, Oxford.

Koul, R. y DANA, T. (1997): Contextualized science for teaching science and technology. Interchange, 28, 121-144.

LAMO, E., GONZÁleZ, J.M. y TORRES, C. (1994): La sociología del conocimiento y de la ciencia. Alianza Editorial, Madrid.

LATOUR, B. y WoOlgar, S. (1996): La vida en el laboratorio. Alianza, Madrid.

LUCAS, A. (1994): STS beyond school: Public perceptions and sources of knowledge. En J. Solomon y G. Aikenhead (Eds.), STS education: International perspectives on reform (pp. 111-119). Teachers College Press, Nueva York.

MERTON, R.K. (1977): La sociología de la ciencia. Alianza Editorial, Madrid.

Miller, J.D. (1983): Scientific literacy: A conceptual and empirical review. Daedalus, 96(1), 29-48.

MilleR, J.D. (1992): Toward a scientific understanding of the public understanding of science and technology. Public Understanding of Science, 1, 23-26.

MitrofF, I. (1974): Norms and counter-norms in a selected group of the Apollo Moon Scientists: A case study of the ambivalence of scientists. American Sociological Review, 39, 579-595.

ReichenBaCh, H. (1966): La filosofia cientifica. FCE, México.

RUBBA, P.A. y HARKNESS, W.L. (1993): Examination of preservice and in-service secondary science teachers' beliefs about Science-Technology-Society interactions. Science Education, 77, 407-431.

VÁzQUeZ, A. y MANASSERO, M.A. (1995): Actitudes relacionadas con la ciencia: una revisión conceptual. Enseñanza de las Ciencias, 13(3), 337-346.

- (1996): Factores determinantes de las actitudes relacionadas con la ciencia. Revista Española de Pedagogía, 203, 43-78.

- Vázquez, A. y Manassero, M.A. (1997): Actitudes y valores relacionados con la ciencia, la tecnología y la sociedad en alumnado y profesorado. Implicaciones para la educación de las actitudes. Memoria final de investigación. MEC-CIDE, Madrid. 